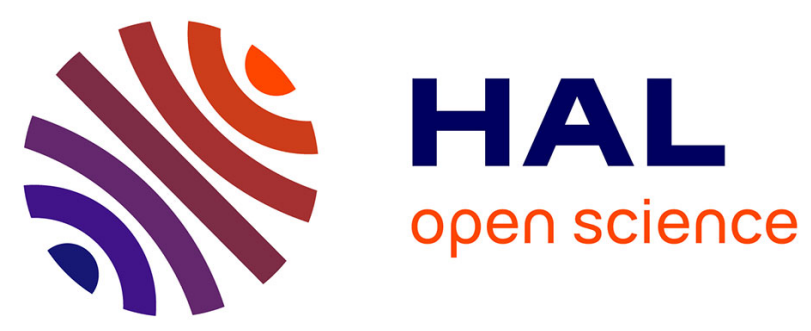

\title{
Flower Patterns in Drop Impact on Thin Liquid Films
}

Guillaume Lagubeau, Marco A Fontelos, Christophe Josserand, Agnès Maurel, Vincent Pagneux, Philippe Petitjeans

\section{To cite this version:}

Guillaume Lagubeau, Marco A Fontelos, Christophe Josserand, Agnès Maurel, Vincent Pagneux, et al. Flower Patterns in Drop Impact on Thin Liquid Films. Physical Review Letters, 2010, 105 (18), 10.1103/PhysRevLett.105.184503 . hal-02454188

\section{HAL Id: hal-02454188 \\ https://hal.science/hal-02454188}

Submitted on 24 Jan 2020

HAL is a multi-disciplinary open access archive for the deposit and dissemination of scientific research documents, whether they are published or not. The documents may come from teaching and research institutions in France or abroad, or from public or private research centers.
L'archive ouverte pluridisciplinaire HAL, est destinée au dépôt et à la diffusion de documents scientifiques de niveau recherche, publiés ou non, émanant des établissements d'enseignement et de recherche français ou étrangers, des laboratoires publics ou privés. 


\title{
Flower Patterns in Drop Impact on Thin Liquid Films
}

\author{
Guillaume Lagubeau, ${ }^{1}$ Marco A. Fontelos, ${ }^{2}$ Christophe Josserand, ${ }^{3}$ Agnès Maurel, ${ }^{4}$ \\ Vincent Pagneux, ${ }^{1}$ and Philippe Petitjeans ${ }^{5}$ \\ ${ }^{1}$ LAUM, UMR CNRS 6613, Avenue Olivier Messiaen, 72085 Le Mans Cedex 9, France \\ ${ }^{2}$ Instituto de Ciencias Matemáticas, (ICMAT, CSIC-UAM-UC3M-UCM), C/ Serrano 123, 28006 Madrid, Spain \\ ${ }^{3}$ Institut D'Alembert, CNRS \& UPMC (Paris 06), UMR 7190, case 162, 4 place Jussieu, 75005 Paris, France \\ ${ }^{4}$ Institut Langevin, LOA, UMR CNRS 7587, ESPCI, 10 rue Vauquelin, 75005 Paris, France \\ ${ }^{5}$ PMMH, UMR CNRS 7636, ESPCI, 10 rue Vauquelin, 75005 Paris, France
}

(Received 15 July 2010; published 27 October 2010)

\begin{abstract}
We describe experimentally the formation of a pattern for drop impacts on thin liquid films for a large range of impact parameters. Using the shallow-water approximation, we are able to explain the main mechanisms leading to these patterns: it consists in the linear instability of the self-similar axisymmetric radial solution of the equations. Agreement between the experiments and the theory is remarkably good, leading, in particular, to the prediction that the most unstable fold number scales like $\left(\mathrm{We} / h_{\infty}\right)^{2 / 7}$.
\end{abstract}

DOI: 10.1103/PhysRevLett.105.184503

PACS numbers: 47.55.D-, 47.20.Ma, 47.55.N-

Introduction.-Drop impact on liquid film is at the heart of our understanding of many complex multiphase flows [1]. It is present in many industrial applications such as spray coating, ink-jet printing or combustion in motor chambers. It is also crucial in environmental contexts, for instance controlling the dissemination of agricultural products [2] or understanding the erosion enhanced by raindrops [3]. It involves a high pressure impulse, rapid velocity changes, strong interface deformations and eventually secondary droplet breakup. In particular, important questions related to drop impact on liquid films concern the splashing transition and the number and the sizes of the secondary droplets eventually ejected by the impact. In general, when a drop hits a thin liquid surface, a crater rapidly expands surrounded by a liquid rim or corolla. The crater corresponds to a thin liquid layer with high velocity field, the corolla is usually unstable and this can lead to the breakup of secondary droplets [4]. When the impact velocity is high enough, the so-called prompt splash can be seen, where a very thin jet and small droplets can be ejected at short times [5-7]. Since the pioneering work of Worthington [8], most studies have focused on the splash formation, particularly for high Weber and Reynolds numbers and thin liquid layers [9-12], as for instance in the well-known pictures of Edgerton [13].

Here, we report on an experimental study of the instability of the corolla for drop impacts on a liquid surface for moderate Weber numbers and thicker liquid layers, where no splash is observed. Drop impact studies have always been strongly linked to the improvement of imaging techniques $[8,14]$. In these footsteps, using a Fourier Transform Profilometry technique (FTP) [15], adapted recently for fluid interface [16-19], we exhibit and analyze for the first time the striking formation of a flowerlike pattern originating from a hydrodynamic instability, illustrated in Fig. 1.

Experiments.-A drop of radius $R_{0}=2.05 \mathrm{~mm}$ is created using a syringe and impacts at velocity $U_{0}$ on a film of the same liquid of thickness $H$ comparable to $R_{0}$. The liquid is water dyed with $0.1 \mathrm{wt} \%$ white pigment supplied by Millenium (surface tension and viscosity remain the same as pure water). The surface deformation was measured by FTP, a technique based on fringe pattern projection on the surface, that allows a resolution of the elevation of the interface with a $50 \mu \mathrm{m}$ accuracy in the three directions each millisecond (for more details see $[16,17]$ ). This technique enables the measurement of the interface for large time after impact; that is to say $t>\tau=R_{0} / U_{0}$ $(\tau \approx 1 \mathrm{~ms})$.

The impact dynamics of droplets is usually governed by the Reynolds and the Weber numbers, defined as:

$$
\operatorname{Re}=\frac{2 R_{0} U_{0}}{\nu} \text { and } \mathrm{We}=\frac{2 \rho R_{0} U_{0}^{2}}{\sigma},
$$

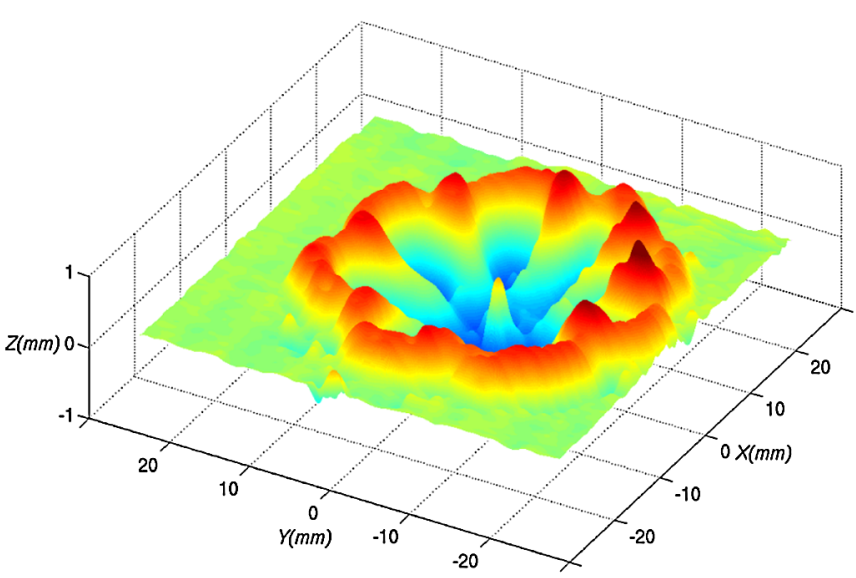

FIG. 1 (color online). Snapshots of the liquid surface deformation $37 \mathrm{~ms}$ after the impact of a water drop $\left(R_{0}=2.05 \mathrm{~mm}\right.$, $\mathrm{We}=136)$ on a water film $(H=3.1 \mathrm{~mm})$. We observe a eightfold flower pattern. The surface deformation was measured using the FTP technique. The liquid is pure water dyed with $0.1 \mathrm{wt} \%$ white pigment. 
where $\rho$ and $\nu$ are, respectively, the liquid density and kinematic viscosity while $\sigma$ is the surface tension. Here, the influence of the surrounding air can be neglected and two additional dimensionless numbers have to be considered: the aspect ratio $R_{0} / H$ and the Froude number $\mathrm{Fr}=U_{0} / \sqrt{g R_{0}}$ that characterizes the importance of the gravity during the impact.

In our experiments, the velocity $U_{0}$ is varied from 1 to $2.8 \mathrm{~m} \cdot \mathrm{s}^{-1}$ by changing the falling height of the drop, and $H$ varied between 1.5 and $3.4 \mathrm{~mm}$. After the impact, we first observe the expansion of a liquid bump of height almost constant in time (Fig. 2, top left and Fig. 3). Later an instability of the bump develops (Fig. 2, top). Eventually the bump collapses and a flower pattern-remanent of the bump instability -is observed temporarily (Fig. 2, bottom left and right, see also supplementary materials for the full movie of the dynamics). Finally, a thick jet is formed at the impact point. We found these dynamics to be robust in the studied parameter range $(4000<\operatorname{Re}<15000,27<$ $\mathrm{We}<222$ and $0.66<H / R_{0}<1.66$ ), and six to 12 -fold symmetry flowers have been observed. We focus here on the first regime, before the bump collapses. A typical height profile measurement is shown on Fig. 3 (bottom) and both the bump position $r$ and thickness $d r$ appear to follow a $\sqrt{t}$ power law (insets of Fig. 4).

Shallow-water approximation.-In the context of the present experiments and for the large enough times $t>\tau$ considered here, the interface can be described by a $2 \mathrm{D}$ mapping $z=h(x, y, t)$. In these conditions, we can use the shallow-water approximation to describe the interface dynamics. Neglecting the viscosity and gravity since
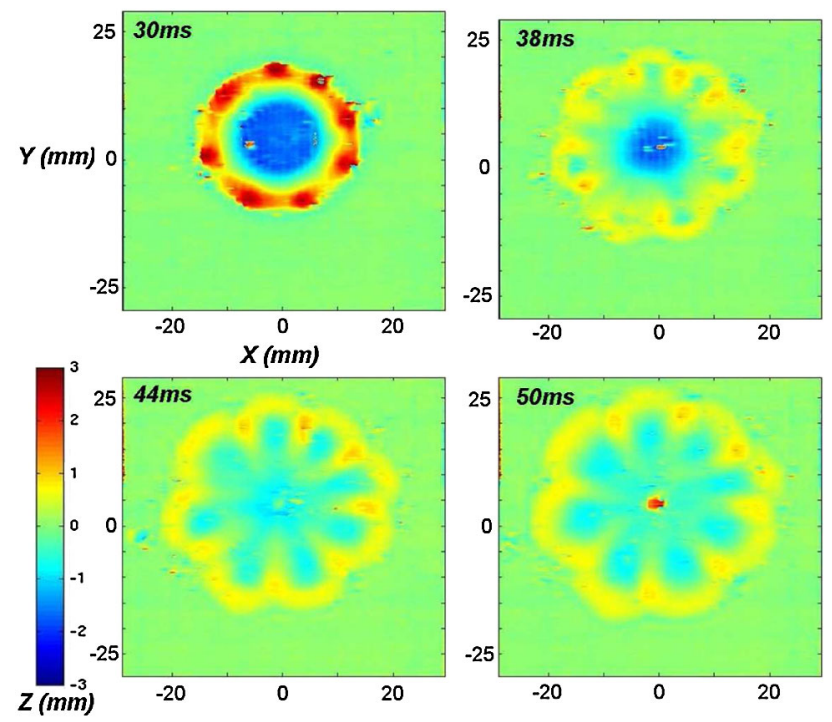

FIG. 2 (color online). Evolution of the liquid interface after a water drop $\left(R_{0}=2.05 \mathrm{~mm}, \mathrm{We}=110\right)$ has impacted a $3.4 \mathrm{~mm}$ deep liquid layer. Snapshots of the surface deformation measured by FTP technique for 30, 38, 44, and $50 \mathrm{~ms}$ after the impact, respectively. The color scale indicates the height of the interface.
$\operatorname{Re}>4000$ and $\mathrm{Fr}>10$ in the experiments, the dynamics is described for radially symmetric solutions by the interface height $h(r, t)$ and the radial velocity $v(r, t)$ :

$$
\begin{aligned}
& \frac{\partial h}{\partial t}+\frac{1}{r} \frac{\partial}{\partial r}(r v h)=0, \\
& \frac{\partial v}{\partial t}+v \frac{\partial v}{\partial r}=\frac{1}{\mathrm{We}} \frac{\partial \kappa}{\partial r},
\end{aligned}
$$

where $\kappa$ is the curvature. For the impact, these equations have been first made dimensionless using the change of variable: $t \rightarrow \frac{U_{0} t}{R_{0}} h \rightarrow \frac{h}{R_{0}} r \rightarrow \frac{r}{R_{0}}$, and we will consider $\varepsilon=\frac{1}{\mathrm{We}} \ll 1$.

The radially symmetric solution can be decomposed in three regions: an inner layer, originating around the region of impact and where the fluid forms a thin film with a more or less constant height; and an outer layer where the film tends asymptotically to a uniform thickness $h_{\infty}=H / R_{0}$. An intermediate region, that can be interpreted as a "shock", matches the inner with the outer region.

Inner layer solution: in the $\varepsilon=0$ limit, the equation for the velocity is Burgers equation. The impact conditions are such that a shock is created in a finite time [4], with explicit solution:

$$
v=\frac{r}{t} \quad \text { and } \quad h=\frac{a}{t^{2}},
$$

where $t$ can be shifted to $t+t_{0}$ and $a$ is a constant. This solution describes the thinning of the film after the complete impact of the drop up to some limiting thickness that is determined by the growth of a viscous boundary layer at its base $[20,21]$.

Outer layer solution: we can seek a solution by means of the self-similar ansatz:
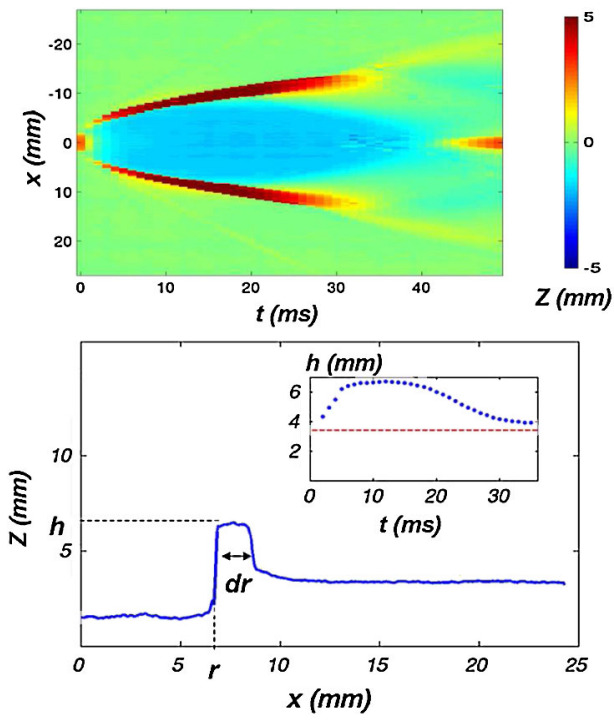

FIG. 3 (color online). Top: spatiotemporal diagram of the height of the interface following a diameter. Bottom: measured bump profile $17 \mathrm{~ms}$ after the impact $(\mathrm{We}=110, H=3.4 \mathrm{~mm})$ ). Inset: maximal height $h$ as a function of time, while the straight line indicates the liquid height at infinity. 


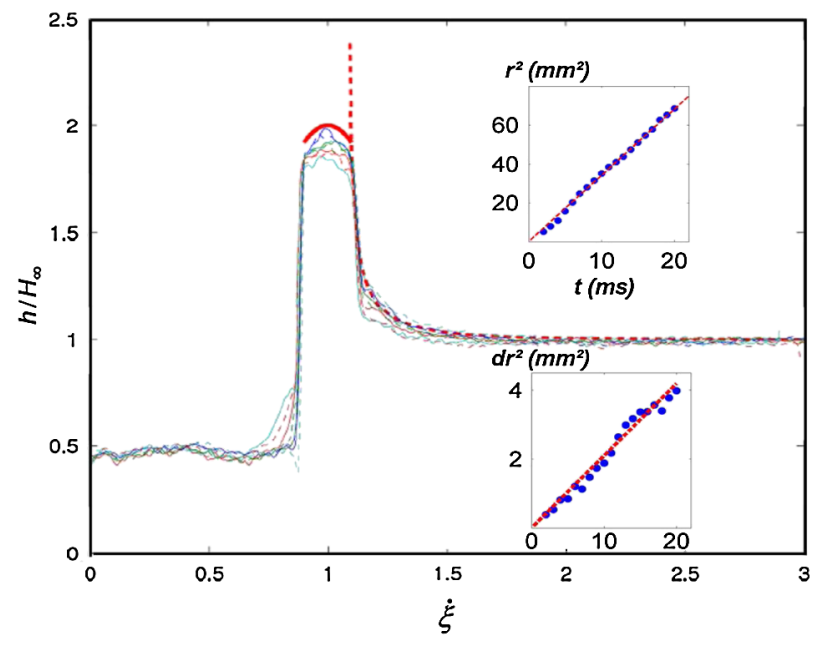

FIG. 4 (color online). Experimental profiles $(\mathrm{We}=110$, $H=3.4 \mathrm{~mm}, t=10$ to $18 \mathrm{~ms}$ ) rescaled with respect to the self-similar structure of the solution according to Eq. (4). Thick dotted red line: theoretical profile for $\mathrm{We}=\infty$ [Eq. (5)]. Thick red line: bump profile given by formula (8) for $\mathrm{We}=110$. Inset top: square of the bump position $(r)$ as a function of time. Inset bottom: square of the bump thickness $(d r)$ as a function of time.

$$
v=\frac{1}{t^{1 / 2}} f\left(\frac{r}{t^{1 / 2}}\right) ; \quad h=h_{\infty} g\left(\frac{r}{t^{1 / 2}}\right) .
$$

The self-similar variable $\xi=r / \sqrt{t}$ is reminiscent of the Burgers equation's structure of Eq. (2) in the limit $\varepsilon=0$ and is in fact common to the inner and the outer solutions. Plugging (4) into Eqs. (1) and (2), we obtain, in the limit $\varepsilon=0$, a system that can be integrated giving:

$$
f(\xi)=\frac{\xi-\sqrt{\xi^{2}-c^{2}}}{2} \text { and } g(\xi)=\frac{\left(\xi+\sqrt{\xi^{2}-c^{2}}\right)^{2}}{4 \xi \sqrt{\xi^{2}-c^{2}}}
$$

where $c$ is a constant, and $g_{\mid \xi \rightarrow c^{+}} \sim \frac{1}{4} \sqrt{\frac{c}{2(\xi-c)}}$ and $g_{\mid \xi \rightarrow \infty} \sim 1+O\left(\xi^{-4}\right)$.

The singularity of $g$ can be understood as a shock separating the inner domain $r \leq c \sqrt{t}$ and the outer domain $r \geq$ $c \sqrt{t}$ where a bump is growing due do the propagation of the shock. Figure 4 shows the experimental profiles rescaled according to (3) for different times, showing the self-similar structure of the expanding bump. The singular behavior of $g$ is found to be in good agreement with the experimental profiles up to the top of the bump where some regularization mechanism has to be invoked. Indeed, it can be found that the leading regularization comes from the surface tension and so by keeping the dominant term $g^{\prime \prime}$ of the curvature for $t \gg 1$, the self-similar structure of the outer solution survives. Balancing the surface tension term with all the others in Eq. (2), we can describe the solution in the form:

$$
v=\frac{1}{t^{1 / 2}} \tilde{f}\left(\eta=\frac{\xi-c}{l_{c}}\right) ; \quad h=h_{\infty} l_{c}^{-(3 / 2)} \tilde{g}(\eta) .
$$

The self-similar scale is $l_{c}=\left(\frac{h_{\infty}}{\mathrm{We}_{\mathrm{e}}}\right)^{2 / 7}$, with

$$
\begin{gathered}
-\frac{1}{2}\left(c+l_{c} \eta\right) \tilde{g}^{\prime}+\frac{1}{c+l_{c} \eta}\left(\left(c+l_{c} \eta\right) \tilde{f} \tilde{g}\right)^{\prime}=0, \\
-\frac{1}{2}\left(\left(c+l_{c} \eta\right) \tilde{f}\right)^{\prime}+\frac{1}{2}\left(\tilde{f}^{2}\right)^{\prime}=-\tilde{g}^{\prime \prime \prime},
\end{gathered}
$$

so that, when $l_{c} \ll 1$, one can integrate Eq. (7) near the bump leading to:

$$
\frac{h}{h_{\infty}} \sim A \mathrm{We}^{3 / 7}-\frac{\mathrm{We}}{16} c^{2}\left(\xi-\xi_{\max }\right)^{2},
$$

where $A$ is, at leading order, independent of the Weber number and $\xi_{\max }$ indicates the position of the top of the bump. On Fig. 4, we represent the experimental profiles rescaled according to Eq. (3) together with the theoretical profile for $\mathrm{We}=\infty$ [Eq. (5)] and with the bump profile [Eq. (8)], at the-finite-experimental Weber number.

To find the global solution of the problem, we need to match the solution of these equations with the inner solutions (3). This can be done by introducing the viscosity in the equations. It has been shown that a viscous boundary layer grows into the inner film solution leading to an asymptotic constant film thickness scaling like $\mathrm{Re}^{-2 / 5} h_{\infty}$ [20,21]. Then, a fairly good approximation of the solution is simply given by considering, in the $t \gg 1$ limit, that the inner solutions satisfy $v=0$ and $h=0$.

In the original parameters, the impact of the drop in this regime leads to a self-similar expanding bump of radius $c \sqrt{U_{0} R_{0} t}$ and thickness $d r \propto l_{c} \sqrt{U_{0} R_{0} t}=\left(\frac{H_{\infty} \gamma}{\rho U_{0}^{2} R_{0}^{2}}\right)^{2 / 7} \times$ $\sqrt{U_{0} R_{0} t}$ with a-time-independent-maximum height $h=H_{\infty}\left(\frac{\rho U_{0}^{2} R_{0}^{2}}{H_{\infty} \gamma}\right)^{3 / 7}$. This situation might be interpreted as a particular hydraulic jump where the gravity is replaced by the inertia stored in the bump [22].

Stability analysis.-In order to describe the appearance of the flower pattern, we study the linear stability of the former radial solution. We use the shallow-water equations in polar coordinates:

$$
\begin{aligned}
& \frac{\partial h}{\partial t}+\frac{1}{r} \frac{\partial}{\partial r}(r v h)+\frac{1}{r} \frac{\partial}{\partial \theta}(r u h)=0, \\
& \frac{\partial v}{\partial t}+v \frac{\partial v}{\partial r}+\frac{u}{r} \frac{\partial v}{\partial \theta}-\frac{u^{2}}{r}=-\frac{1}{\mathrm{We}} \frac{\partial \kappa}{\partial r} \\
& \frac{\partial u}{\partial t}+v \frac{\partial u}{\partial r}+\frac{u}{r} \frac{\partial u}{\partial \theta}+\frac{u v}{r}=-\frac{1}{\mathrm{We}} \frac{1}{r} \frac{\partial \kappa}{\partial \theta}
\end{aligned}
$$

where $u$ is the azimuthal velocity. Denoting $\left(\tilde{f}_{0}, \tilde{g}_{0}\right)$ the extension of the radial solution $(\tilde{f}, \tilde{g})$ towards the inner region, we can develop the azimuthal stability analysis following the same scalings: $h(r, \theta, t)=h_{\infty} l_{c}^{-(3 / 2)}\left(\tilde{g}_{0}(\eta)+\right.$ $\left.\tilde{g}_{1}(\eta, \theta, \tau)\right), \quad v(r, \theta, t)=t^{-(1 / 2)}\left(\tilde{f}_{0}(\eta)+\tilde{f}_{1}(\eta, \theta, \tau)\right)$, $u(r, \theta, t)=t^{-(1 / 2)} \tilde{q}_{1}(\eta, \theta, \tau)$. For the perturbed analysis, the pertinent time scale is $\tau=\ln (t)$. By decomposing $\left(\tilde{g}_{1}, \tilde{f}_{1}, \tilde{q}_{1}\right)$ in angular modes $\left(\tilde{G}_{1}, \tilde{F}_{1}, \tilde{Q}_{1}\right) e^{\omega_{n} \tau+i n \theta}$ and assuming that the eigenmodes are concentrated near the 


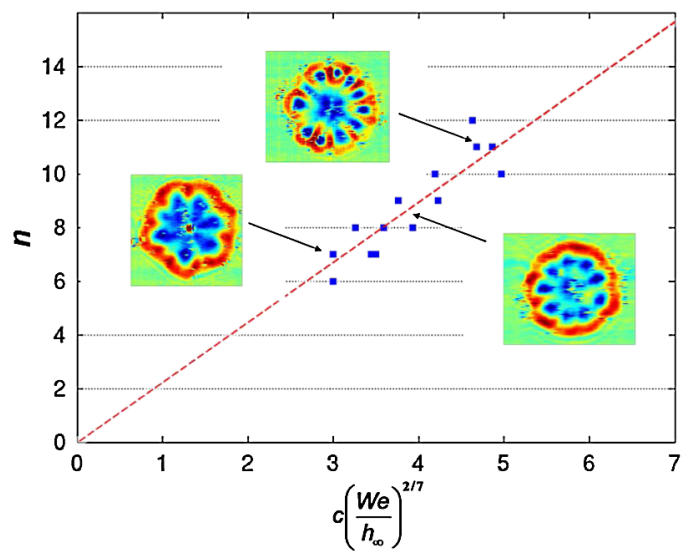

FIG. 5 (color online). Flower mode number $n$ as a function of the parameter $c\left(\frac{\mathrm{We}}{h_{\infty}}\right)^{2 / 7}$. Inserts: experimental observations using the FTP technique for $n=7,9$ and 11. The color scale indicates the height of the interface, increasing from blue $(-0.75 \mathrm{~mm})$ to red $(0.75 \mathrm{~mm})$. Relative errors in the horizontal measures are below $7 \%$ while the vertical error is \pm 0.5 .

bump $(\eta \sim 0)$, we obtain at leading order $\left(l_{c} \ll 1\right)$ the simplified relations

$l_{c} \omega_{n} \tilde{G}_{1}-\frac{1}{2} c \frac{d \tilde{G}_{1}}{d \eta}+\frac{d}{d \eta}\left(\tilde{g}_{0} \tilde{F}_{1}+\tilde{f}_{0} \tilde{G}_{1}\right)+\frac{i n l_{c}}{c} \tilde{g}_{0} \tilde{Q}_{1}=0$,

$l_{c} \omega_{n} \tilde{F}_{1}-\frac{c}{2} \frac{d \tilde{F}_{1}}{d \eta}+\tilde{f}_{0} \frac{d \tilde{F}_{1}}{d \eta}+\tilde{F}_{1} \frac{d \tilde{f}_{0}}{d \eta}=-\left(\frac{d^{2}}{d \eta^{2}}-\frac{n^{2} l_{c}^{2}}{c^{2}}\right) \frac{d \tilde{G}_{1}}{d \eta}$,

$l_{c} \omega_{n} \tilde{Q}_{1}-\frac{c}{2} \frac{d \tilde{Q}_{1}}{d \eta}+\tilde{f}_{0} \frac{d \tilde{Q}_{1}}{d \eta}=-\frac{i n l_{c}}{c}\left(\frac{d^{2}}{d \eta^{2}}-\frac{n^{2} l_{c}^{2}}{c^{2}}\right) \tilde{G}_{1}$.

This linear system depends only on three coefficients so that we have an implicit relation $l_{c} \omega_{n}=\mathcal{F}\left(n l_{c} / c, c\right)$. Therefore, without solving the linear system explicitly and taking into account the slight dependence of $c$ with the Weber number, we argue that the fold index of the maximum growth rate scales like

$$
n_{\max } \propto \frac{c}{l_{c}}=c\left(\frac{\mathrm{We}}{h_{\infty}}\right)^{2 / 7} .
$$

The index of the unstable modes was measured in the different experiments carried out and it agrees remarkably well with the predicted theoretical scaling as shown Fig. 5. Finally, can one relate this instability to some physical mechanism? Two familiar surface instability candidates are the Rayleigh-Taylor (RT) or Rayleigh-Plateau (RP) instabilities. RT would act differently on the inner and outer sides of the bump. In the frame of reference attached to the bump the inner side would be stable and the outer unstable since the bump is decelerating. But the inner side of the shock covers a higher area so that this is an unlikely source of instability. On the other hand, since the bump can be viewed approximately as half a torus that is fixed in the self-similar frame, we expect the RP instability to be the leading instability mechanism, in agreement with the origi- nal scenario of droplet breakup drawn by Worthington [23]. The amplitude growth of the perturbations can discriminate between these two instabilities: one expects a $\tau^{2} \sim(\ln (t))^{2}$ evolution for RT and a $e^{\gamma \tau} \sim t^{\gamma t}$ for RP. Preliminary results (see supplementary materials [24]) suggest an algebraic growth of the instability in agreement with RP.

Finally, it is tempting to try to link this self-similar dynamics to the question of secondary droplet formation. Indeed, we would like to emphasize that the higher the Weber number and the smaller $h_{\infty}$, then the higher and the thinner the self-similar bump is, suggesting eventually droplet breakup.

M. A. Fontelos acknowledges the project MTM200803255 for its support, C. Josserand the financial support of the Agence Nationale de la Recherche through grant ANR-09-JCJC-0022 (ANR Deformation), and G. Lagubeau, A. Maurel, V. Pagneux, and P. Petitjeans the financial support of the Agence Nationale de la Recherche through grant ANR-08-BLAN-0108 (ANR Tourbillonde).

[1] A. L. Yarin, Annu. Rev. Fluid Mech. 38, 159 (2006).

[2] V. Bergeron, D. Bonn, J.-Y. Martin, and L. Vovelle, Nature (London) 405, 772 (2000).

[3] O. Planchon and E. Mouche, Soil Sci. Soc. Am. J. 74, 1092 (2010).

[4] A. Yarin and D. Weiss, J. Fluid Mech. 283, 141 (1995).

[5] S. Thoroddsen, J. Fluid Mech. 451, 373 (2002).

[6] C. Josserand and S. Zaleski, Phys. Fluids 15, 1650 (2003).

[7] R. D. Deegan, P. Brunet, and J. Eggers, Nonlinearity 21, C1 (2008).

[8] A. Worthington, Proc. R. Soc. London 25, 261 (1876).

[9] A.-B. Wang and C.-C. Chen, Phys. Fluids 12, 2155 (2000).

[10] I. Roisman and C. Tropea, J. Fluid Mech. 472, 373 (2002).

[11] S. T. Thoroddsen, T. G. Etoh, and K. Takehara, J. Fluid Mech. 478, 125 (2003).

[12] I. Roisman, K. Horvat, and C. Tropea, Phys. Fluids 18, 102104 (2006).

[13] H. Edgerton and J. Killian, Flash (Branford, Boston, 1954).

[14] S. Thoroddsen, T. Etoh, and K. Takehara, Annu. Rev. Fluid Mech. 40, 257 (2008).

[15] M. Takeda and K. Mutoh, Appl. Opt. 22, 3977 (1983).

[16] P. Cobelli, A. Maurel, V. Pagneux, and P. Petitjeans, Exp. Fluids 46, 1037 (2009).

[17] A. Maurel, P. Cobelli, V. Pagneux, and P. Petitjeans, Appl. Opt. 48, 380 (2009).

[18] P. Cobelli, V. Pagneux, A. Maurel, and P. Petitjeans, Europhys. Lett. 88, 20006 (2009).

[19] P. Cobelli, P. Petitjeans, A. Maurel, V. Pagneux, and N. Mordant, Phys. Rev. Lett. 103, 204301 (2009).

[20] I. Roisman, Phys. Fluids 21, 052104 (2009).

[21] J. Eggers, M. Fontelos, C. Josserand, and S. Zaleski, Phys. Fluids 22, 062101 (2010).

[22] T. Bohr, P. Dimon, and V. Putkaradze, J. Fluid Mech. 254, 635 (1993).

[23] A. Worthington, Proc. R. Soc. London 30, 49 (1879).

[24] See supplementary material at http://link.aps.org/ supplemental/10.1103/PhysRevLett.105.184503. 